\title{
Spectrum of fast dynamics in glass forming liquids: Does the "knee" exist?
}

J. Gapiński, W. Steffen, A. Patkowski, A. P. Sokolov, A. Kisliuk, U. Buchenau, M. Russina, F. Mezei, and H. Schober

Citation: The Journal of Chemical Physics 110, 2312 (1999); doi: 10.1063/1.477966

View online: https://doi.org/10.1063/1.477966

View Table of Contents: http://aip.scitation.org/toc/jcp/110/5

Published by the American Institute of Physics

\section{Articles you may be interested in}

Hyper-resolution Brillouin-Rayleigh spectroscopy with an optical beating technique Review of Scientific Instruments 64, 2136 (1993); 10.1063/1.1143950

Mechanisms of resonant low frequency Raman scattering from metallic nanoparticle Lamb modes The Journal of Chemical Physics 146, 194201 (2017); 10.1063/1.4983119

Including surface ligand effects in continuum elastic models of nanocrystal vibrations

The Journal of Chemical Physics 147, 044711 (2017); 10.1063/1.4995439

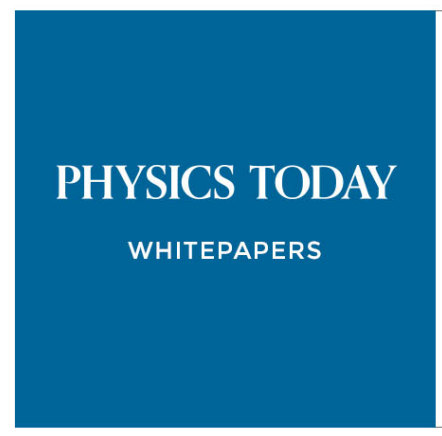




\title{
Spectrum of fast dynamics in glass forming liquids: Does the "knee" exist?
}

\author{
J. Gapiński, ${ }^{\text {a) }}$ W. Steffen, and A. Patkowski ${ }^{\text {a) }}$ \\ Max-Planck-Institut für Polymerforschung, 55128 Mainz, Germany
}

A. P. Sokolov
Institut für Festkörperforschung, Forschungszentrum Jülich, 52425 Jülich, Germany

A. Kisliuk

Institut für Experimentalphysik, FU Berlin, 14195 Berlin, Germany

U. Buchenau

Institut für Festkörperforschung, Forschungszentrum Jülich, 52425 Jülich, Germany

M. Russina and F. Mezeib)

Hahn-Meitner-Institut, Glieniker Str. 100, 14109 Berlin, Germany

H. Schober

ILL, Avenue des Martyrs, BP156X, 38190 Grenoble, France

(Received 18 August 1998; accepted 25 November 1998)

\begin{abstract}
A knee-shaped feature observed earlier in light scattering spectra of $\mathrm{Ca}_{0.4} \mathrm{~K}_{0.3}\left(\mathrm{NO}_{3}\right)_{1.4}(\mathrm{CKN})$ below $T_{c}$ is used as a strong argument in favor of mode-coupling theory of the glass transition (MCT). Our careful measurements reveal no "knee" in the spectra of two glass forming liquids, CKN and ortho-terphenyl. Instead of the knee the spectra show nontrivial broadening and an increase of the intensity with a temperature increase. Both variations are confirmed by neutron scattering measurements on CKN and are neither expected in the asymptotic MCT predictions nor in any other model. (C) 1999 American Institute of Physics. [S0021-9606(99)51605-X]
\end{abstract}

Recent developments of the mode coupling theory $(\mathrm{MCT})^{1}$ stimulated extensive experimental studies of the glass transition. MCT predicts the existence of a crossover temperature $T_{c}$ with different scenarios for dynamics of glass forming systems above and below. Quasielastic neutron $(\mathrm{QENS})^{2,3}$ and light ${ }^{4-6}$ scattering measurements, and also computer simulations, ${ }^{7}$ demonstrated reasonable agreement with MCT predictions for the high-temperature range above $T_{c} . T_{c}$ was estimated to be higher than the conventional glass transition temperature $T_{g}$. The dynamics of glass forming liquids in the temperature range below $T_{c}$ was not investigated so extensively. Here MCT predicts a nontrivial behavior of the generalized susceptibility, $\chi^{\prime \prime}(\nu): \chi^{\prime \prime}(\nu) \propto \nu$ at lower frequencies (white-noise spectrum) and $\chi^{\prime \prime}(\nu) \propto \nu^{a}$, a $<0.4$ at higher frequencies. This behavior results in a "knee" in $\chi^{\prime \prime}(\nu)$ at intermediate frequencies.

According to MCT, the main variation of the fast dynamics spectrum at $T<T_{c}$ should be a shift of the knee to lower frequencies with increasing temperature. The characteristic scaling time of the knee, $\tau_{\beta}=1 / 2 \pi \nu_{\beta}$ ( $\nu_{\beta}$ is the position of the knee) should diverge at $T_{c}$. This scenario is schematically presented in Fig. 1. The existence of the knee with a nontrivial temperature dependence is a crucial point in testing the asymptotic MCT predictions below $T_{c}$. An analysis of experimental data, however, reveals some inconsistency: the knee was reported in depolarized light scattering

\footnotetext{
a) Also at the Institute of Physics, A. Mickiewicz University, Poznan, Poland.

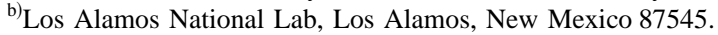

spectra of $\mathrm{Ca}_{0.4} \mathrm{~K}_{0.3}\left(\mathrm{NO}_{3}\right)_{1.4}(\mathrm{CKN})$, and its temperature variation was found to be in a good agreement with the MCT predictions; ${ }^{4}$ no such agreement was observed in depolarized light scattering spectra of ortho-terphenyl (OTP). ${ }^{5}$ No knee was reported in $\mathrm{QENS}^{2}$ and dielectric spectroscopy ${ }^{8}$ studies of CKN. Also, recent light scattering data obtained for CKN at temperatures below $T_{g}$ demonstrated the absence of the knee. ${ }^{9}$

Thus, the existence of the knee appears to be a crucial question at present. The nontrivial prediction for the temperature behavior of the knee, e.g., a divergence of the time scale with increasing temperature at $T_{c}$, is counterintuitive for dynamics of supercooled liquids and was the subject of intensive discussions in the literature. ${ }^{10}$ The knee observed in the light scattering spectra of $\mathrm{CKN}$ is considered as a strong support in favor of the MCT scenario. It is our main goal in this Communication to answer this question on the basis of careful depolarized light and neutron scattering studies of glass forming liquids between $T_{c}$ and $T_{g}$.

Samples of CKN $\left(T_{g} \approx 333 \mathrm{~K}, T_{c} \approx 370-380 \mathrm{~K}^{2,4}\right)$ and OTP $\left(T_{g} \approx 244 \mathrm{~K}, T_{c} \approx 290 \mathrm{~K}^{5}\right)$ were prepared, as described earlier. ${ }^{5,11}$ Depolarized light scattering spectra in the frequency range $0.5-300 \mathrm{GHz}$ were measured using a Sandercock 6-pass tandem Fabry-Perot interferometer (STFPI) in right-angle geometry and an $\mathrm{Ar}^{+}$laser $(\lambda=514.5 \mathrm{~nm})$. QENS of CKN has been done on the time-of-flight spectrometer IN5 at ILL (Grenoble) using neutrons with the wavelength $5 \AA$. The spectra were corrected in a standard way. 


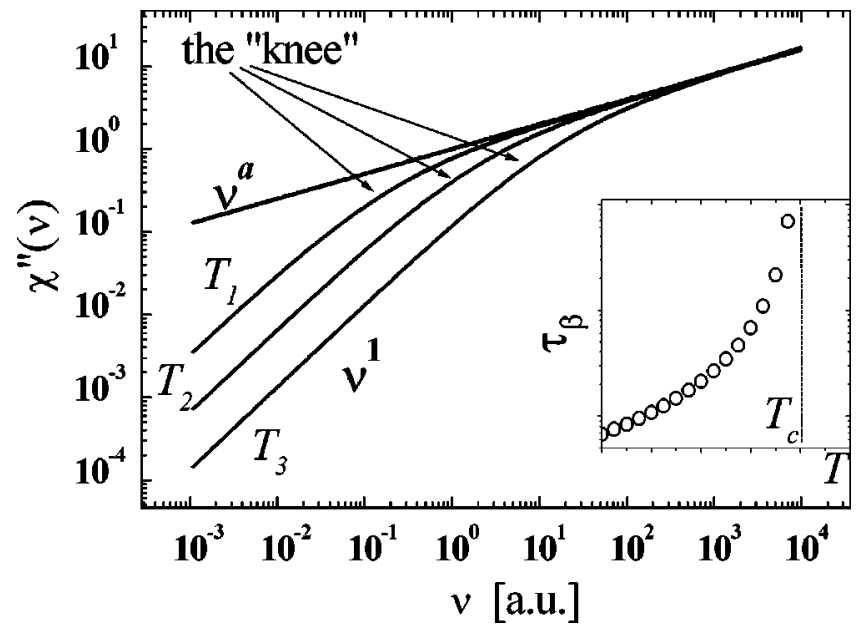

FIG. 1. A schematic representation of the MCT scenario for generalized susceptibility at temperatures below $T_{c}, T_{c}>T_{1}>T_{2}>T_{3}$. The knee corresponds to a transition from $\nu^{1}$ to $\nu^{a}$ behavior. The inset shows the predicted temperature dependence of the inverse frequency of the knee $\tau_{\beta}$.

Details of the QENS measurements will be presented elsewhere. ${ }^{12}$

In order to understand how a knee can occur in STFPI measurements, we have to discuss the technique in some detail. Transmission spectra of the Fabry-Perot interferometer have sharp maxima at constant frequency intervals, called the free spectral range (FSR). As a result, a measured spectrum is a superposition (sum) of many spectra (orders) shifted by one FSR with respect to each other. A great advantage of the tandem setup designed by Sandercock (two interferometers arranged in a special geometry ${ }^{13}$ ) is the suppression of the nearest 19 neighboring orders on either side of the central peak. However, in the case of very broad spectra covering several decades of frequency, the contribution of every 20th order, for which the transmission of a STFPI is again very good (enhanced orders), may become important. The natural bandwidth $B(\mathrm{THz})$ of the STFPI, defined by a set of a pinhole, a lens, and a prism, ranges from 2 to $15 \mathrm{THz}$ (half-width), depending on the pinhole size $d(\mathrm{~mm}): B$ $=15 d^{13}$. The number of enhanced orders falling into the tandem window for small FSRs can be as high as 18 (Table I). This fact is usually neglected by tandem users.

In order to suppress the higher orders of the spectrum, we used two narrow interference filters: half-width at halfmaximum $(\mathrm{HWHM}) \approx 600 \mathrm{GHz}$ and $\approx 70 \mathrm{GHz}$, respectively. The first filter was used to measure the spectra at FSR $=150$ and $35 \mathrm{GHz}$, and the second one at FSR $=10 \mathrm{GHz}$. The spectra were corrected for the transmission function (measured using white light from a lamp) of the STFPI with the filters. Raman spectra obtained earlier ${ }^{5,11}$ were used at higher frequencies.

TABLE I. The maximum number of transmitted STFPI spectra for different FSR (in GHz) and the pinhole size (in $\mathrm{mm}$ ).

\begin{tabular}{llllr}
\hline \hline FSR & 6 & 30 & 50 & 300 \\
pinhole & 0.15 & 0.3 & 0.45 & 1 \\
$\mathrm{~N}_{20}$ & 18 & 7 & 6 & 2 \\
\hline \hline
\end{tabular}

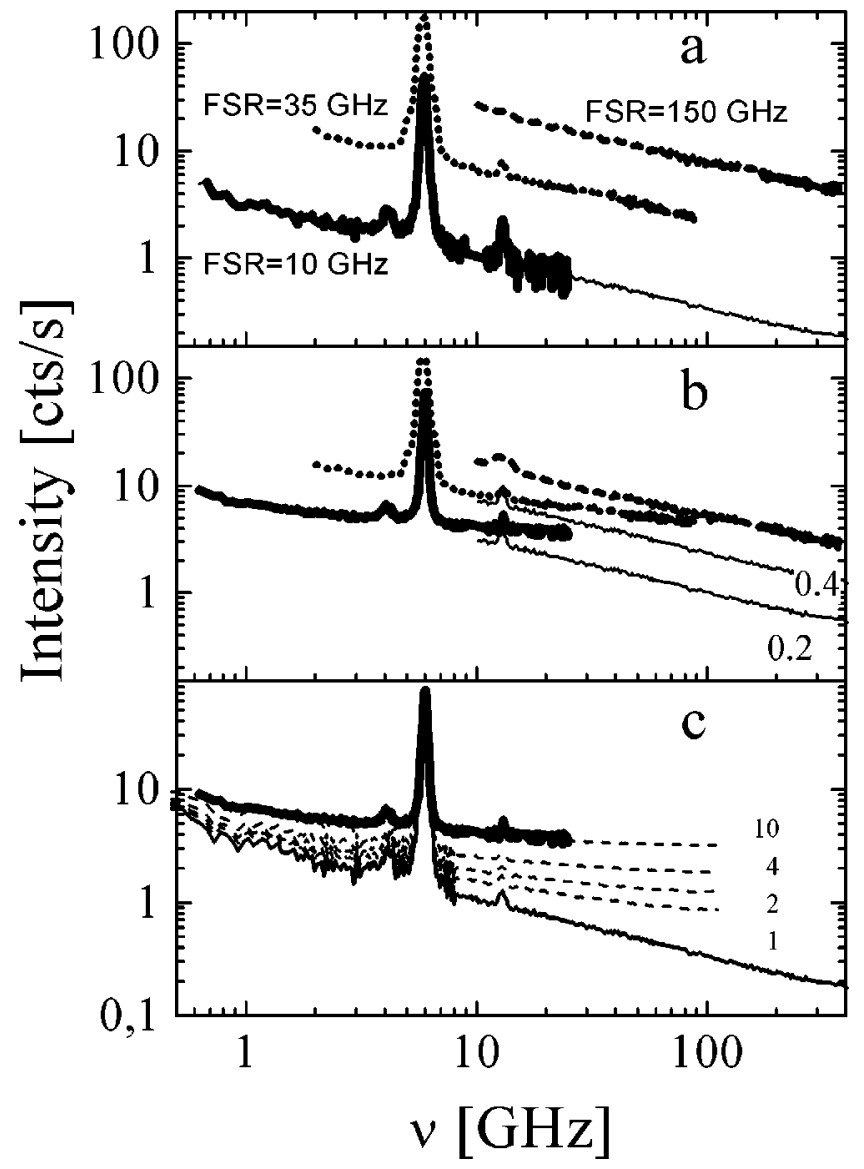

FIG. 2. The spectra of $C K N(T=337 \mathrm{~K})$ with (a) and without (b) filters. $\mathrm{FSR}=150 \mathrm{GHz}$ (dashed), $35 \mathrm{GHz}$ (dotted), and $10 \mathrm{GHz}$ (thick solid line) as indicated in (a). The thin solid line in (a): the spectrum at $\mathrm{FSR}=150 \mathrm{GHz}$ scaled by 0.05 , by $0.4,0.2$ in (b). (b) depending on the scaling factor, one can get the "knee" at any frequency between 10 and $100 \mathrm{GHz}$. (c) A combined spectrum (with filter, solid line), simulated spectra that include 1, 2, 4 and 10 orders (dashed lines).

Figure 2 presents spectra of $\mathrm{CKN}$ at $T=337 \mathrm{~K}$ measured with different FSR and corrected for the transmission function. The spectra measured with the interference filters show a similar shape at different FSR [Fig. 2(a)], whereas the spectra measured without the filters differ strongly, even in the frequency range where they overlap [Fig. 2(b)]. One also notes that the spectra measured with small FSR without the filters have higher intensity than the ones measured with the filters. Intensity measured at different FSR should decrease at least as much as the FSR itself. In the case of the measurements with the filters [Fig. 2(a)], the intensity decreases about 20 times when FSR is changed about 15 times, from 150 to $10 \mathrm{GHz}$. However, in the case of the measurements without the filters, the intensity decreases only about four times for the same changes of FSR [Fig. 2(b)]. This result points out that there is some additional contribution to the spectrum measured without the filters.

In order to estimate the influence of the higher-order contributions of the STFPI on the measured spectrum we simulated additional contributions, using the spectrum measured with the filters [Fig. 2(c)]. The simulated spectrum that takes into account the first \pm 10 transmitted orders reproduces well the spectrum measured without the filter. These 


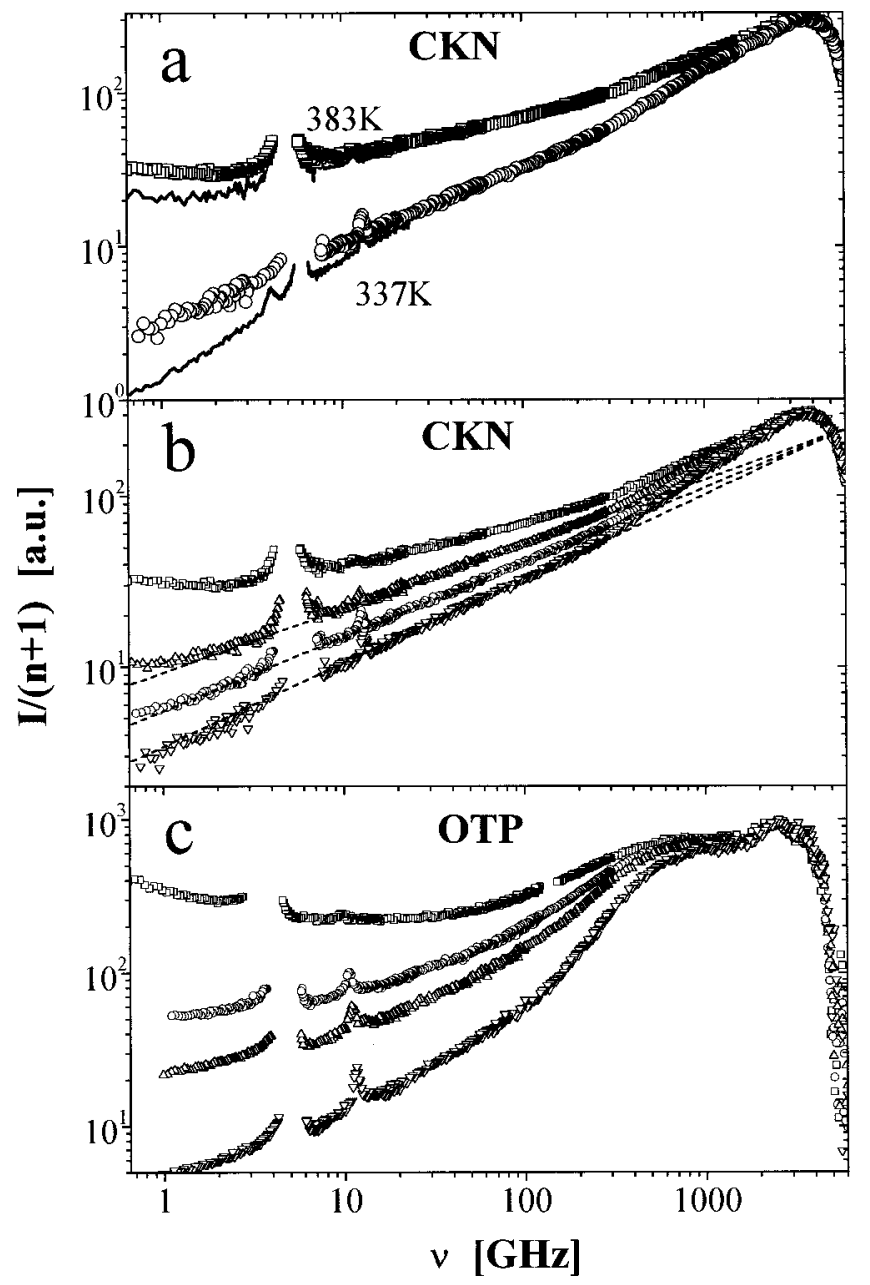

FIG. 3. (a) Spectra of CKN at two temperatures combined from the measurements performed with (symbols), without (solid lines) the filters. (b) Spectra of CKN measured at 337, 350, 362, $383 \mathrm{~K}$ (from bottom to top), and (c) spectra of OTP measured at 240, 260, 270, $290 \mathrm{~K}$ (from bottom to top). Spectra in (b) and (c) were measured with the filters. Dashed lines in (b) show power law dependencies.

results [Fig. 2(c)] give a clear explanation of the difference between the spectra measured at small FSR with and without the filter. An important point to note: the spectrum measured without the filter is much flatter than the one measured with the filter. The reason is clear: the spectra of the higher orders are nearly constant in the frequency range of the central order and their contributions can be considered as an additional flat background.

Figure 3(a) presents a comparison of the susceptibility spectra, $\chi^{\prime \prime}(\nu)=I /(n+1)\left(\right.$ here $n+1=[\exp (-h \nu / k T)-1]^{-1}$ is the Bose factor for the energy loss side), measured with and without the filters. As it is clear from Fig. 2, the spectra measured without filters have higher intensities than the ones measured with filters. However, the former appear to be below the latter after a scaling to the Raman data due to the different scaling factors [Fig. 3(a)]. At low temperatures one can clearly see the knee in the spectrum measured without filters. The position of the knee is arbitrary and depends on the matching procedure [Fig. 2(b)]. However, the spectrum measured with the filters shows no knee and can be well approximated by a single power law. One also notes a dif-

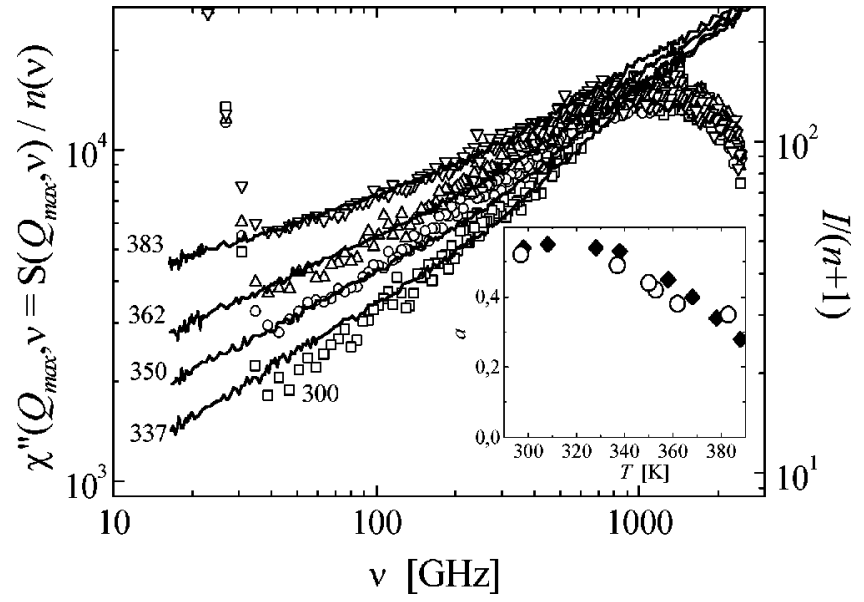

FIG. 4. CKN: the neutron scattering susceptibility (symbols) measured around the main diffraction maximum $\mathrm{Q}_{\max }=1.9 \AA^{-1}$ (integrated in the range $1.5-2.25 \AA^{-1}, n(\nu)=[\exp (h \nu / k T)-1]^{-1}$ temperature factor on energy win side, no corrections for Debye-Waller factor, more detailed description will be presented in Ref. 12). Lines show the light scattering susceptibility spectra. One should pay attention that the lowest temperatures differ: $T=300 \mathrm{~K}$ for neutrons and $T=337 \mathrm{~K}$ for the light scattering. The inset shows the temperature variation of the exponent a: $(\bigcirc)$ our data $(\downarrow)$, Ref. 15.

ference in the spectra measured at higher temperatures: the minimum between the fast dynamics and a slow $\alpha$ process is also affected by the higher orders. In that case, however, the difference is rather quantitative while at low temperatures we have a qualitative difference in the spectral shape.

Figures 3(b) and 3(c) present the susceptibility spectra of CKN and OTP, measured with the filters at different temperatures. No sign of the knee can be seen in the spectra. The spectra of CKN at temperatures below $360 \mathrm{~K}$ can be well approximated by a power law, $\chi^{\prime \prime}(\nu) \propto \nu^{\mathrm{a}}$, with the exponent a increasing with decreasing temperature. In the case of OTP the spectra do not show a single power-law shape and the slope decreases with decreasing frequency, i.e., the spectra are curved in the direction opposite to the expected knee. Whether this is due to peculiarities of the fast process, or due to a contribution of some slower process (the so-called, slow $\beta$ relaxation, which is known to be strong in dielectric spectra of OTP, or the "fast" process in the $\mathrm{GHz}$ frequency range suggested earlier ${ }^{5}$ ) is not clear from our data. One can see, however, that the slope of the OTP spectrum becomes steeper with decreasing temperature [Fig. 3(c)] in the same way as for CKN.

Figure 3 also demonstrates that the quasielastic intensity at high frequencies decreases strongly with decreasing temperature between $T_{c}$ and $T_{g}$. This result is inconsistent with the asymptotic MCT predictions (Fig. 1). It was also reported in Ref. 4 and was interpreted as a peculiar behavior of the light scattering mechanism (the cancellation effect for the dipole-induced-dipole mechanism). However, neutron scattering spectra of CKN demonstrate the same variations for the quantity directly related to density fluctuations (the subject of the MCT's equations) (Fig. 4). Moreover, a direct comparison of the data (Fig. 4) shows that both light scattering and QENS spectra have very similar spectral shape and its temperature variations (the difference of the spectra at 
$\nu>1000 \mathrm{GHz}$ is related to the vibrational contribution, which usually has a frequency-dependent coupling coefficient $^{14}$ ). Obviously, the decrease of the intensity with decreasing temperature is mainly an intrinsic property of the fast process and is not a specific property of the light scattering mechanism. The results suggest an explanation for the temperature variation of the so-called nonergodicity parameter. The latter is related to an integrated intensity of the fast process and its temperature variation was observed in many neutron scattering experiments. According to MCT, these variations should be related to the shift of the knee (Fig. 1). Our results (Figs. 3 and 4) demonstrate, however, that the reason for the variation is the change of the amplitude of the fast process.

An important nontrivial observation is a decrease of the exponent a with a temperature increase (inset Fig. 4). For $\mathrm{CKN}$ it agrees reasonably well with the earlier data presented by Tao, Li, and Cummins. ${ }^{15}$ However, according to the asymptotic MCT predictions, the exponent a should be temperature independent. The power-law spectral shape for the fast relaxation, $\chi^{\prime \prime}(\nu) \propto \nu^{a}$, is also predicted in the model of asymmetric double-well potentials. ${ }^{16}$ In that case the exponent a should increase with temperature, $\mathbf{a} \propto T$, approaching $\mathbf{a}=1$ at high temperatures. That has been recently observed, in particular, for CKN at temperatures below $T_{g} \cdot{ }^{9}$ Our results demonstrate an opposite trend at temperatures above $T_{g}$ : the fast relaxation spectra of CKN and OTP become more stretched (the exponent a decreases) with an increase of temperature. We do not see any clear explanation for that nontrivial observation, neither in MCT nor in any other model description.

As a conclusion, our results (Figs. 2 and 3) demonstrate that there is no knee in the depolarized light scattering spectra of CKN and OTP and suggest that the knee observed earlier in $\mathrm{CKN}^{4}$ is an instrumental artifact due to additional contributions coming from the higher orders of the STFPI used (the authors did not use interference filters). The reason for the knee is clear: a flat background from higher orders [see Fig. 2(c)] gives in the susceptibility spectrum a contribution with $\chi^{\prime \prime}(\nu) \propto I^{*} \nu / T \propto \nu$, dominating the low-intensity spectra measured without the filters at low frequencies and finally producing the artificial knee. One also can explain an apparent temperature shift of the artificial knee: the quasielastic intensity at low frequencies decreases with temperature much stronger than the intensity at higher frequencies (Fig. 3). As a result, the ratio of the real spectrum to the flat background from the higher orders decreases and the artificial knee shifts to higher frequencies with a decreasing temperature. Thus, the asymptotic scenario suggested by MCT for the temperature range below $T_{c}$ (Fig. 1) disagrees with the presented experimental data, even qualitatively: (i) there is no knee in the spectra, (ii) the main variation is a decrease of the intensity and change of the slope of the spectra with decreasing temperature (Figs. 3 and 4). One certainly needs more experimental data, especially neutron scattering measurements extended to lower frequency in order to shed more light on this problem.

Partial financial support of the Deutsche Forschungsgemeinschaft (SFB 262) is gratefully acknowledged.

${ }^{1}$ W. Götze and L. Sjögren, Rep. Prog. Phys. 55, 241 (1992).

${ }^{2}$ W. Knaak, F. Mezei, and B. Farago, Europhys. Lett. 7, 429 (1988).

${ }^{3}$ M. Kiebel et al., Phys. Rev. B 45, 10301 (1992); W. Petry and J. Wuttke, Transp. Theory Stat. Phys. 24, 1075 (1995).

${ }^{4}$ G. Li, W. M. Du, X. K. Chen, H. Z. Cummins, and N. J. Tao, Phys. Rev. A 45, 3867 (1992); H. Z. Cummins, W. M. Du, M. Fuchs, W. Götze, S. Hildebrand, A. Latz, G. Li, and N. J. Tao, Phys. Rev. E 47, 4223 (1993).

${ }^{5}$ W. Steffen, A. Patkowski, H. Gläser, G. Meier, and E. W. Fischer, Phys. Rev. E 49, 2992 (1994)

${ }^{6}$ J. Wuttke et al., Phys. Rev. Lett. 72, 3052 (1994); A. Brodin et al., Phys. Rev. B 53, 11511 (1996).

${ }^{7}$ W. Kob, in Annual Reviews of Computational Physics, edited by D. Stauffer (World Scientific, Singapore, 1995), Vol. III, p. 1.

${ }^{8}$ P. Lunkenheimer, A. Pimenov, and A. Loidl, Phys. Rev. Lett. 78, 2995 (1997).

${ }^{9}$ N. V. Surovtsev, J. Wiedersich, V. N. Novikov, E. Rössler, and A. P. Sokolov (submitted)

${ }^{10}$ X. C. Zeng, D. Kivelson, and G. Tarjus, Phys. Rev. E 50, 1711 (1994); H. Z. Cummins and G. Li, ibid. 50, 1720 (1994).

${ }^{11}$ A. P. Sokolov et al., Phys. Rev. Lett. 71, 2062 (1993); J. Non-Cryst. Solids 172-174, 138 (1994).

${ }^{12}$ A. P. Sokolov et al. (in preparation).

${ }^{13}$ Manual instruction for Sandercock tandem interferometer, etc.; J. R. Sandercock, Top. Appl. Phys. 51, 173 (1982).

${ }^{14}$ A. P. Sokolov, U. Buchenau, W. Steffen, B. Frick, and A. Wischnewski, Phys. Rev. B 52, R9815 (1995).

${ }^{15}$ N. J. Tao, G. Li, and H. Z. Cummins, Phys. Rev. Lett. 66, 1334 (1991).

${ }^{16}$ K. S. Gilroy and W. A. Phillips, Philos. Mag. B 43, 735 (1981). 